\title{
COMMENT \\ Nutrition and the developing brain
}

\author{
Christina J. Valentine ${ }^{1}$ \\ Pediatric Research (2020) 87:190-191; https://doi.org/10.1038/s41390-019-0650-y
}

The first thousand days from conception to 2 years is a critical period for nutrient interventions to impact lifelong health, ${ }^{1}$ particularly development. ${ }^{2}$ Exponential brain growth in both volume and mapping of neuronal pathways are occurring during this timeframe, ${ }^{3}$ and thus prioritizing nutrients that can maximize neural health and development, such as calories, protein, fatty acids, iron, zinc, iodine, and choline, specifically becomes a priority, ${ }^{4}$ particularly for the vulnerable preterm infant who misses tremendous nutrition over the last trimester. ${ }^{5}$ It can also offer opportunity to improve already injured areas of the brain in term infants by increasing total enteral protein intake. ${ }^{6}$ New magnetic resonance imaging (MRI) techniques offer a non-invasive window to observe specific areas of the brain associated with motor, cognitive, and behavior function ${ }^{7}$ even after injury and may offer predictive, biomarker applicability. ${ }^{8}$ Observational and intervention studies demonstrate a clear relationship between nutrient intake or the "lack of" on the synthesis and function of neurotransmitters, neural membranes, energy metabolism membrane components, and synapses all impacting development not only for cognition but also behavior and mood. ${ }^{9,10}$ Focusing on the nutrient quantity and quality is therefore important. In fact, new pre-clinical studies connect the relevance of iron nutrition to gene expression in the brain and subsequent effects. ${ }^{11}$ Consequently, inadequate iron in early infancy impacts behavior, development, and is even associated with schizophrenia later in life. ${ }^{10}$ In addition, sophisticated MRI functional examinations corroborate the lack of similar brain development in the preterm counterparts when compared to the term counterparts and stress the importance of early calories and lipids. ${ }^{7}$ More specific attention to not only total lipid but clearly the long-chain polyunsaturated fatty acids not only need to be provided at certain doses but also their ratio matters for development and behavioral outcomes, ${ }^{12}$ executive function, ${ }^{13}$ improved word reading and anxiety in children with attention deficit and hyperactivity disorders, ${ }^{14}$ and in mood or depressive disorders. ${ }^{15}$ Confounding the effect of nutrition specifically influencing brain mechanisms is the mounting evidence that demonstrates that both undernutrition and overnutrition also impact the commensal pattern of microbes, which secondarily influences vitamin, shortchain fatty acid production, satiety, and behavior, and thus developmental outcomes independently. ${ }^{16}$ What is critical therefore when doing systematic reviews or developing a meta-analysis to examine "optimal nutrition" is to define author statements of "adequate", "high," or "low"-as nutrient doses are frequently not harmonized across studies being examined, the delivery of these nutrients, often in different formats, such as supplements, various types of human milk, or formulas, can directly affect the brain and the microbiome. More collaborative, prospective research trials should be done to really understand the blend of nutrition that should be achieved. In this issue, the paper, Nutrition and the developing brain: the road to optimizing early neurodevelopment: $a$ systematic review describes systematic review findings that early nutrition matters particularly with mother's milk and adequate calories, but brings out the disparity in results with protein and long-chain polyunsaturated fatty acids. ${ }^{17}$ The review was well conducted, but out of 594 studies only 22 met the stringent criteria to avoid bias and enhance reliability. It was pointed out by the authors that not only the nutrients studied were variable, but the time period study crossed differences in feeding practices now in the United States, such as human milk and donated human milk. Specifically, when determining human milk's effect on term and preterm MRI findings, there were differences depending on the study examined. MRI results were enhanced in term and preterm babies with mother's own milk, ${ }^{18,19}$ however not when a mixture of donor milk:mother's milk was included. ${ }^{20}$ Studies should delineate donor milk separately from mother's own milk as it has both decreased nutritional and bioactive values. ${ }^{21}$ Lactational stage can be as late as 6-12 months and therefore fall short on key nutrients for growth and development. ${ }^{22}$ This may in part explain the differences in the trials examined and a recent trial that demonstrated a higher incidence of infants with lower Bayley Mental development exam scores $<85$ in very low birth weight infants randomized to donor milk as compared to formula. ${ }^{23}$ The authors wish to point out that the maturational findings on MRI clearly demonstrate the superiority of maternal milk feeding for the first 28 days in low birth weight infants $<30$ weeks early on, but lose significance at long-term follow-up $i^{19}$ however, cognitive testing remained significant. In their review, ${ }^{17}$ they did not observe specific protein effects on brain development; however it has recently been described that fat-free mass, weight gain, and body mass index are associated with brain size in preterm infants but not fat mass, ${ }^{24}$ thus stressing the importance of a balanced ratio of calories, lipids, and proteins needed to achieve lean growth. The mixed results on omega 3 in this series highlights the importance of docosahexaenoic acid:arachidonic acid (DHA:AA) ratio, supplementation dose, and duration. For example, the lowdose trial by van Wezel-Meijler et al. ${ }^{25}$ was a nicely executed, double-blind, randomized controlled trial (RCT) with DHA and AA, given in formula at $0.015 \mathrm{~g} / 100 \mathrm{ml} \mathrm{DHA}$ and $0.031 \mathrm{~g} / 100 \mathrm{ml} \mathrm{AA}$, respectively, as compared to a formula without DHA/AA, and found no difference in brain structure; however, while the ratio of DHA:AA was on par with the recommendations, the total doses were well below the recommendations; ${ }^{26}$ thus, surprisingly, no results were obtained. In contrast, the omega 3 used in Almaas et al. ${ }^{27}$ was a follow-up of a previous RCT using very high-dose liquid supplement of $0.86 \% \mathrm{DHA}$ and $0.91 \% \mathrm{AA}$ given in addition

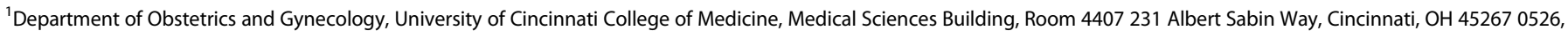
USA

Correspondence: Christina J. Valentine (Christina.valentine@rb.com)

Received: 9 September 2019 Revised: 22 October 2019 Accepted: 23 October 2019

Published online: 31 October 2019 
to the feeding for weeks $1-9 .^{28}$ The resulting 1:1 ratio and significantly higher DHA than used in previous successful formula studies on development $(0.32 \% \mathrm{DHA} / 0.64 \% \mathrm{AA})$ may be the explanation for the reduction in results on development. ${ }^{12}$ Reviewing the previous detail in the follow-up trial by Almaas et al. ${ }^{27}$ infants had a significant improvement in DHA plasma levels but experienced a decrease in $A A$ plasma, ${ }^{28}$ thus emphasizing the importance of balance for fatty acid experiments. AA plays a key role in brain maturation in addition to DHA and also is accreted exponentially in the last trimester; ${ }^{29}$ thus, attention to infant adequacy to both fatty acids is crucial. In fact, a recent $R C T$ in pregnant women with adequate DHA:AA resulted in increased brain volume of their term infants using MRI scans. ${ }^{30}$ Focus beyond just calories and protein should start to include specific fatty acids. In addition, future examinations of human milk-fed infants should differentiate the donor milk component and emphasize what fortification was used. Finally, the insights provided by this excellent review prompt the consideration of serial imaging of the brain, standardized measurements of growth along with optimizing nutrient interventions ${ }^{17}$ to further our understanding on early interventions and lifelong health.

\section{ADDITIONAL INFORMATION}

Competing interests: Dr. Valentine is an employee of Mead Johnson Nutrition, but developed this under the academic nature of her University position.

Publisher's note Springer Nature remains neutral with regard to jurisdictional claims in published maps and institutional affiliations.

\section{REFERENCES}

1. Schwarzenberg, S. J. \& Georgieff, M. K., Committee on Nutrition. Advocacy for improving nutrition in the first 1000 days to support childhood development and adult health. Pediatrics 114, 68-77 (2018).

2. Rosales, F. J., Reznick, J. S. \& Zeisel, S. H. Understanding the role of nutrition in the brain and behavioral development of toddlers and preschool children. Nutr. Neurosci. 12, 190-202 (2009).

3. Bluml, S., Wisnowski, J. L. \& Panigrahy, A. Metabolic maturation of the human brain from birth through adolescence: insights from in vivo magnetic resonance spectroscopy. Cereb. Cortex 23, 2944-2955 (2013).

4. Georgieff, M. K., Ramel, S. E. \& Cusick, S. E. Nutritional influences on brain development. Acta Pediatr. 107, 1310-21. (2018).

5. Ehrenkranz, R. A. Nutrition, growth and clinical outcomes. World Rev. Nutr. Diet. 110, 11-26 (2014).

6. Merhar, S. L. et al. Safety and tolerability of enteral protein supplementation for infants with brain injury. Nutr. Clin. Pract. 30, 546-50. (2015).

7. Schneider, J. et al. Nutrient intake in the first two weeks of life and brain growth in preterm neonates. Pediatrics 141, e20172169 (2018).

8. Georgieff, M. K., Krebs, N. F. \& Cusick, S. E. The benefits and risks of iron supplementation in pregnancy and childhood. Ann. Rev. Nutr. 39, 121-146 (2019).

9. Lozoff, B. et al. Long lasting neural and behavior effects of iron deficiency in infancy. Nutr. Rev. 64, 534-591. (2006).
10. Ennis, K. et al. Early life iron deficiency alters glucose transporter 1 expression in the adult rodent hippocampus. J. Nutr. 149, 1660-1666 (2019).

11. Hammerl, M. et al. Reduced cerebellar size at term equivalent age is related to a $17 \%$ lower mental developmental index in very preterm infants without brain injury. Neonatology 3, 1-8 (2019).

12. Colombo, J. et al. Docosahexaenoic acid (DHA) and arachidonic acid (AA) balance in developmental outcomes. Prostaglandins Leukot. Essent. Fatty Acids 121, 52-56 (2017).

13. Adjepong, M. et al. Whole blood $n-3$ fatty acids are associated with executive function in 2-6 year-old Northern Ghanaian children. J. Nutr. Biochem. 57, 287-93. (2018).

14. Milte, C. M. et al. Polyunsaturated fatty acids, cognition and literacy in children with ADHD with and without learning difficulties. J. Child Health Care 15, 299-311 (2011).

15. McNamara, R. K., Vannest, J. J. \& Valentine, C. J. Role of perinatal long-chain omega-3 fatty acids in cortical circuit maturation: mechanisms and implications for psychopathology. World J. Psychiatry 5, 15-34 (2015).

16. Gomez-Gallegro, C. et al. The microbiota and malnutrition: impact of nutritional status during early life. Annu. Rev. Nutr. 39, 267-90. (2019).

17. Ottolini, K. M., Andescavage, N., Keller, S. \& Limperopoulos, C. Nutrition and the developing brain: the road to optimizing early neurodevelopment: a systematic review. Pediatr. Res. (2019). https://doi.org/10.1038/S41390-019-0508-3. PMID 31349359.

18. Isaacs, E. B. et al. Impact of breastmilk on intelligence quotient, brain size, and white matter development. Pediatr. Res. 67, 357-362 (2010).

19. Belfort, M. B. et al. Breast milk feeding, brain development, and neurocognitive outcome: a 7 year longitudinal follow up study in infants born less than 30 weeks gestation. J. Pediatr. 177, 133-39. e.1 (2016).

20. Vasu, V. et al. Preterm nutritional intake and MRI phenotype at term age: a prospective observational study. BMJ Open 4, e005390 (2014).

21. Ballard, O. \& Morrow, A. L. Human milk composition:nutrients and bioactive factors. Peditr. Clin. N. Am. 60, 49-74 (2013).

22. Valentine, C. J. et al. Lactational stage of pasteurized human donor milk contributes to nutrient limitations for infants. Nutrients 9, pii. E302 (2017). https://doi. org/10.3390/nu9030302. PMID 28335478.

23. O'Connor, D. L. et al. Effect of supplemental donor human milk compared with preterm formula on neurodevelopment of very low birth weight infants at 18 months: a randomized clinical trial. JAMA 316, 1897-1905 (2016).

24. Bell, K. A. et al. Associations of growth and body composition with brain size in preterm infants. J. Pediatr., 214, 20-26 (2019).

25. van Wezel-Meijler, G. et al. Dietary supplementation of long-chain polyunsaturated fatty acids in preterm infants: effects on cerebral maturation. Acta Paediatr. 91, 942-950 (2002).

26. Robinson, D. T. \& Martin, C. R. Fatty acid requirements for the preterm infant Semin. Fetal Neonatal Med. 22, 8-14 (2017).

27. Almaas, A. N. et al. Diffusion tensor imaging and behavior in premature infants at 8 years of age, a randomized controlled trial with long-chain polyunsaturated fatty acids. Early Hum. Dev. 95, 41-46 (2016).

28. Henriksen, C. et al. Improved cognitive development among preterm infants attributable to early supplementation of human milk with docosahexaenoic acid and arachidonic acid. Pediatrics 121, 1137-1145 (2008).

29. Alessandri, J. M. et al. Polyunsaturated fatty acids in the CNS evolution of concepts and nutritional implications throughout life. Reprod. Nutr. Dev. 44, 509-38. (2004).

30. Ogundipe, E. et al. Randomized controlled trial of brain specific fatty acid supplementation in pregnant women increases brain volume in MRI scans of their newborn infants. Prostaglandins Leukot. Essent. Fatty Acids 138, 6-13 (2018). 